\title{
Strong spin-phonon coupling in infrared and Raman spectra of $\mathrm{SrMnO}_{3}$
}

\author{
S. Kamba,, , * V. Goian,${ }^{1}$ V. Skoromets,${ }^{1}$ J. Hejtmánek, ${ }^{1}$ V. Bovtun,${ }^{1}$ M. Kempa, ${ }^{1}$ \\ F. Borodavka, ${ }^{1}$ P. Vaněk, ${ }^{1}$ A.A. Belik, ${ }^{2}$ J.H. Lee, ${ }^{3}$ O. Pacherová, ${ }^{1}$ and K.M. Rabe ${ }^{3}$ \\ ${ }^{1}$ Institute of Physics ASCR, Na Slovance 2, 18221 Prague 8, Czech Republic \\ ${ }^{2}$ International Center for Materials Nanoarchitectonics (WPI-MANA), \\ National Institute for Materials Science (NIMS), 1-1 Namiki, Tsukuba, Ibaraki 305-0044, Japan \\ ${ }^{3}$ Department of Physics and Astronomy, Rutgers University, Piscataway, New Jersey 08854-8019, USA
}

(Dated: May 26, 2022)

\begin{abstract}
Infrared reflectivity spectra of cubic $\mathrm{SrMnO}_{3}$ ceramics reveal $18 \%$ stiffening of the lowest-frequency phonon below the antiferromagnetic phase transition occurring at $\mathrm{T}_{N}=233 \mathrm{~K}$. Such a large temperature change of the polar phonon frequency is extraordinary and we attribute it to an exceptionally strong spin-phonon coupling in this material. This is consistent with our prediction from first principles calculations. Moreover, polar phonons become Raman active below $\mathrm{T}_{N}$, although their activation is forbidden by symmetry in $P m \overline{3} m$ space group. This gives evidence that the cubic $P m \overline{3} m$ symmetry is locally broken below $\mathrm{T}_{N}$ due to a strong magnetoelectric coupling. Multiphonon and multimagnon scattering is also observed in Raman spectra. Microwave and $\mathrm{THz}$ permittivity is strongly influenced by hopping electronic conductivity, which is caused by small non-stoichiometry of the sample. Thermoelectric measurements show room-temperature concentration of free carriers $n_{e}=3.610^{20} \mathrm{~cm}^{-3}$ and the sample composition $\mathrm{Sr}^{2+} \mathrm{Mn}_{0.98}^{4+} \mathrm{Mn}_{0.02}^{3+} \mathrm{O}_{2.99}^{2-}$. The conductivity exhibits very unusual temperature behavior: $\mathrm{THz}$ conductivity increases on cooling, while the static conductivity markedly decreases on cooling. We attribute this to different conductivity of the ceramic grains and grain boundaries.
\end{abstract}

PACS numbers: 75.80.+q; 78.30.-j; 63.20.-e

\section{INTRODUCTION}

In the last decade, there has been an intensive search for new multiferroic materials, in particular those exhibiting ferroelectric (FE) and ferromagnetic (FM) order. The aim is to find multiferroics with a large magnetoelectric coupling, which could allow the control of magnetization by external electric field. Such materials could be used in future electronic devices including non-volatile memories and spin filters. Unfortunately, most multiferroics exhibit antiferromagnetic (AFM) order with a weak magnetoelectric coupling or low critical temperatures, and thus they are not suitable for practical applications.

One mechanism that has been demonstrated to produce novel multiferroics is application of an epitaxial strain (or a comparable perturbation) to an AFM paraelectric material with a large spin-phonon coupling such that the lowest frequency polar phonon with hypothetical ferromagnetic (FM) ordering has lower frequency than the corresponding phonon in the AFM phase. Lowering of the polar phonon frequency by the strain can produce a polar instability in the FM phase, with the resulting energy lowering, which stabilizes a FE-FM multiferroic phase over the bulk AFM paraelectric phase $\stackrel{1}{\underline{m}}$ This has been experimentally confirmed in $\mathrm{EuTiO}_{3}$ with a magnetic ordering temperature of $5.3 \mathrm{~K}^{2}$

Recently, Lee and Rabe showed using first-principles calculations that this mechanism would produce a multiferroic phase in $\mathrm{SrMnO}_{3} \cdot \frac{3}{2}$ Since bulk $\mathrm{SrMnO}_{3}$ is AFM below $\mathrm{T}_{N}=230 \mathrm{~K}, 4$ strained thin films should be FM with critical temperatures above 100 or $150 \mathrm{~K} \stackrel{3}{*}$ How- ever, due to the very high critical strain (3.6\%), experimental efforts to realize this phase have been so far unsuccessful. Tuning of the polar phonons by a related but different perturbation, negative chemical pressure through continuous substitution of $\mathrm{Sr}$ by Ba, has recently been investigated. Sakai et al $\underline{5}, 6_{6}$ observed ferroelectricity below $410 \mathrm{~K}$ in $\mathrm{Sr}_{1-x} \mathrm{Ba}_{x} \mathrm{MnO}_{3}$ single crystals with $0.45 \leq x \leq 0.50$. The polar distortion is found to be dominated by displacement of magnetic $\mathrm{Mn}^{4+}$ cations (running counter to the conventional expectation that $d$ electrons inhibit off-centering of the cations ${ }^{7}$ ) and therefore the magnetoelectric coupling is the largest attained so far $\frac{5}{-}$ However, the magnetic ordering for the ferroelectric phase is G-type AFM, so that ferroelectricity is the result of destabilization of the polar mode by strain within the original AFM phase, and the system is not a true (FM-FE) multiferroic.

The prospects for stabilizing a true multiferroic in $\mathrm{SrMnO}_{3}$ or related compounds hinge on better knowledge and control of the spin-phonon coupling. Fortunately, the spin-phonon coupling can be directly investigated experimentally. Specifically, this coupling should result in a remarkable stiffening of the Slater-type (i.e. vibration of Mn cations against oxygen octahedra) mode frequency near and below $\mathrm{T}_{N}$. phonon frequency at $\mathrm{T}_{N}$ have recently been observed in $\mathrm{Sr}_{1-x} \mathrm{Ba}_{x} \mathrm{MnO}_{3}$ for $\mathrm{x}=0-0.3, \frac{6}{\underline{\underline{n}}}$ explaining the observed reduction in the $\mathrm{FE}$ distortion in the AFM phase. In this paper, we investigate the spin-phonon coupling and related properties through detailed $\mathrm{THz}$, far infrared (FIR) and Raman spectroscopic studies of $\mathrm{SrMnO}_{3}$ and combine them with thorough investigations of transport and 
dielectric properties.

\section{EXPERIMENTAL DETAILS}

The tolerance factor of $\mathrm{SrMnO}_{3}$ is very close to unity $(\tau=1.05)$ and therefore it can be synthesized in both cubic and hexagonal crystal structures depending on the growing procedure $\underline{\underline{9}}$ We initially prepared the hexagonal $\mathrm{SrMnO}_{3}$ powder from a stoichiometric mixture of $\mathrm{SrCO}_{3}$ and $\mathrm{Mn}_{2} \mathrm{O}_{3}$ by annealing in air at $1150{ }^{\circ} \mathrm{C}$ for $50 \mathrm{~h}$ and at $1000{ }^{\circ} \mathrm{C}$ for $24 \mathrm{~h}$. Then we pelletized the hexagonal $\mathrm{SrMnO}_{3}$ with a small amount of PVA and annealed at $1000{ }^{\circ} \mathrm{C}$ for $55 \mathrm{~h}$. Afterwards, pellets of the single-phase cubic $\mathrm{SrMnO}_{3}$ were prepared in two steps: $\stackrel{10}{\underline{10}}$ First, we annealed the pellets of hexagonal $4 \mathrm{H}-\mathrm{SrMnO}_{3}$ at $1500{ }^{\circ} \mathrm{C}$ for 8 hours in an Ar flow with the heating/cooling rate of $300{ }^{\circ} \mathrm{C} / \mathrm{min}$. The weight loss after the first step gave the composition of $\mathrm{SrMnO}_{2.53(2)}$. During the second step, we oxidized the obtained $\mathrm{SrMnO}_{2.53}$ sample in air at $350{ }^{\circ} \mathrm{C}$ for $15 \mathrm{~h}$. The weight gain after the second step gave the composition of $\mathrm{SrMnO}_{2.99(2)}$. The density of the ceramics was measured to be $5.50(2) \mathrm{g} / \mathrm{cm}^{3}$; it is about $97.0 \%$ of the powder density, and about $95.8 \%$ of the theoretical XRD density.

The phase purity of the cubic $\mathrm{SrMnO}_{3}$ ceramics was checked by x-ray diffraction and no contamination by the hexagonal phase or by other second phases was discovered. We used the diffractometer Bruker D8 DISCOVER equipped with rotating $\mathrm{Cu}$ anode $[\lambda(\mathrm{CuK \alpha} 1)=1.540598$ $\AA ; \lambda(\mathrm{CuK \alpha 2})=1.544426 \AA$ ] operating with $12 \mathrm{~kW}$ power. A parabolic Göbel mirror on the side of the incident beam and Soller collimator and 200-LiF analyzator on the side of the diffracted beam were used. The crystal structure was measured between 170 and $303 \mathrm{~K}$. The temperature was controlled by a cooling stage Anton Paar DCS 350 and no change of the crystal structure with temperature was observed. The lattice parameter $a_{0}$ determined from 220 and 310 Bragg diffractions linearly changed with temperature from $3.7988(3)$ at $170 \mathrm{~K}$ to $3.8062(4) \AA$ at $303 \mathrm{~K}$. We have to stress here, that we observed no anomaly in $a_{0}(\mathrm{~T})$ around $\mathrm{T}_{N}$.

The temperature of the magnetic phase transition was checked using calorimetry. The ceramic pellet was placed in an aluminum pan and measured using a differential scanning calorimeter Perkin Elmer Pyris Diamond DSC. The sample was cooled from $300 \mathrm{~K}$ down to $100 \mathrm{~K}$ with a cooling rate of $10 \mathrm{~K} / \mathrm{min}$. The same rate was used for heating. An anomaly at $233 \mathrm{~K}$ corresponding to the AFM phase transition was observed in both cooling and heating curves without any temperature hysteresis (see Fig. (1).

The spectroscopic experiments were performed using a Fourier-transform infrared (IR) spectrometer Bruker IFS 113v equipped with a helium-cooled bolometer (operating temperature $1.6 \mathrm{~K}$ ) as a detector and a custommade time-domain $\mathrm{THz}$ spectrometer $\stackrel{11}{\underline{1}}$ In both experiments, Optistat CF cryostats (Oxford Instruments) with

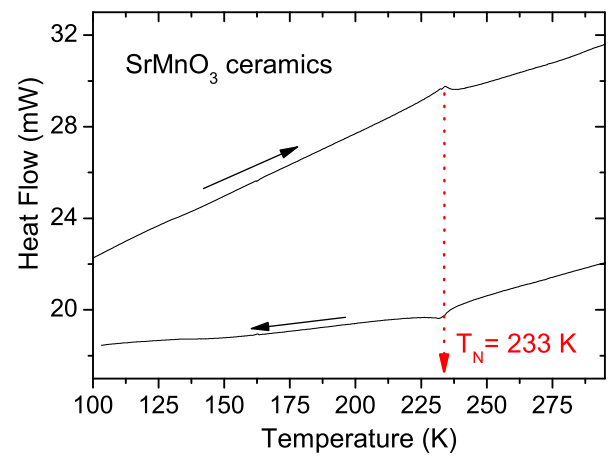

FIG. 1: (Color online) Heat flow measured on cooling and heating. It shows an anomaly near the antiferromagnetic phase transition at $\mathrm{T}_{N}=233 \mathrm{~K}$ without any thermal hysteresis.

polyethylene (far IR) or Mylar (THz) windows were used for measurements in the $10-300 \mathrm{~K}$ temperature range. In the THz spectrometer, a femtosecond Ti:sapphire laser oscillator (Coherent, Mira) produces a train of femtosecond pulses which generates linearly polarized broadband THz pulses in a photoconducting switch TeraSED (GigaOptics). A gated detection scheme based on electro-optic sampling with a $1 \mathrm{~mm}$-thick [110] ZnTe crystal as a sensor allows us to measure the time profile of the electric field of the transmitted $\mathrm{THz}$ pulse (see Ref. 11 for further details).

Ceramic pellets were carefully polished using diamond paste in order to obtain a flat surface with an optical quality. For IR studies, the sample was glued on a circular metallic aperture with a diameter of $5 \mathrm{~mm}$. The sample IR reflectivity was normalized by the reflectivity of a gold mirror sputtered on polished glass, which was glued on the similar aperture. An optical cryostat allowed us to move the sample holder at all temperatures, so we have measured the sample and reference spectra at each temperature. For the time-domain $\mathrm{THz}$ transmission experiments we prepared a polished plane-parallel plate of ceramics with thickness of $50 \mu \mathrm{m}$.

IR reflectivity and $\mathrm{THz}$ complex permittivity spectra were carefully fitted assuming the dielectric function in the form of sum of classical three-parameter damped oscillators 12

$$
\varepsilon^{*}(\omega)=\varepsilon_{\infty}+\sum_{j=1}^{n} \frac{\Delta \varepsilon_{j} \omega_{T O j}^{2}}{\omega_{T O j}^{2}-\omega^{2}+\mathrm{i} \omega \gamma_{T O j}},
$$

where $\omega_{T O j}, \gamma_{T O j}$ and $\Delta \varepsilon_{j}$ denote the eigenfrequency, damping and dielectric strength of the $j$-th transversal optic (TO) polar phonon. $\varepsilon^{*}(\omega)$ is related to the reflectivity $R(\omega)$ of the bulk sample by

$$
R(\omega)=\left|\frac{\sqrt{\varepsilon^{*}(\omega)}-1}{\sqrt{\varepsilon^{*}(\omega)}+1}\right|^{2} .
$$

The high-frequency permittivity $\varepsilon_{\infty}=7.6$ given by electronic absorption processes was obtained from 
the room-temperature frequency-independent reflectivity tails above the phonon frequencies. The temperature dependence of this quantity was neglected, consistent with its behavior in other related perovskite dielectrics $\underline{\underline{13}}$

For Raman studies, a Renishaw RM 1000 MicroRaman spectrometer equipped with a CCD detector and a Linkam THMS 600 temperature cell was used. The experiments were performed in a backscattering geometry in the $100-2000 \mathrm{~cm}^{-1}$ range. He-Ne laser with the wavelength $633 \mathrm{~nm}$ was used.

The magnetic susceptibility data were collected with a Quantum Design (MPMS) superconducting quantum interference device when the applied magnetic field was 100 Oe. The thermoelectric power and thermal conductivity measurements were carried out between 3.5 and $320 \mathrm{~K}$ using a close-cycle refrigerator. The four-point steady-state method was applied in order to eliminate thermal resistances between sink and heater; the electrical contacts were realized using a silver paint. The experimental setup was checked using reference samples. For more details we refer to Ref 14 .

Dielectric measurements in the frequency range from $1 \mathrm{MHz}$ to $1.8 \mathrm{GHz}$ were performed using a computercontrolled dielectric spectrometer equipped with an Agilent 4291B Impedance analyzer, Novocontrol BDS 2100 coaxial sample cell, and Sigma System M18 temperature chamber (operating range of $100-500 \mathrm{~K}$ ). The dielectric parameters were calculated taking into account the electromagnetic field distribution in the sample.

\section{CALCULATIONAL DETAILS}

First-principles calculations were performed using density-functional theory within the generalized gradient approximation GGA $+U$ method with the PerdewBecke-Erzenhof parametrization as implemented in the Vienna ab initio simulation package (VASP-4.6). Details are described in Ref. 8 . The phonon frequencies depend on the choice of the lattice constant and the value of onsite Coulomb interaction $U_{\text {eff }}$. With $U_{\text {eff }}=2.3 \mathrm{eV}$, the zero-temperature phonon frequencies computed for antiferromagnetic spin ordering at $a_{0}=3.825 \AA$ intermediate between the computed lattice constant and the theoretical lattice constant, are in good agreement with the lowtemperature experimental values. We note a previous first-principles calculation of phonons at the experimental lattice constant $a_{0}=3.806 \AA$ yielded good agreement for the TO2 frequency $\frac{18}{}$; however, this is not inconsistent as the latter calculations did not include spin polarization. The temperature dependent spin correlation $\left(\left\langle\mathrm{S}_{i} \cdot \mathrm{S}_{j}\right\rangle\right)$ was calculated using mean-field theory with the exchange constant computed at $a_{0}=3.825 \AA$ and from this the temperature dependent phonon frequencies are obtained using the spin-phonon parameterization of Ref. 1 .
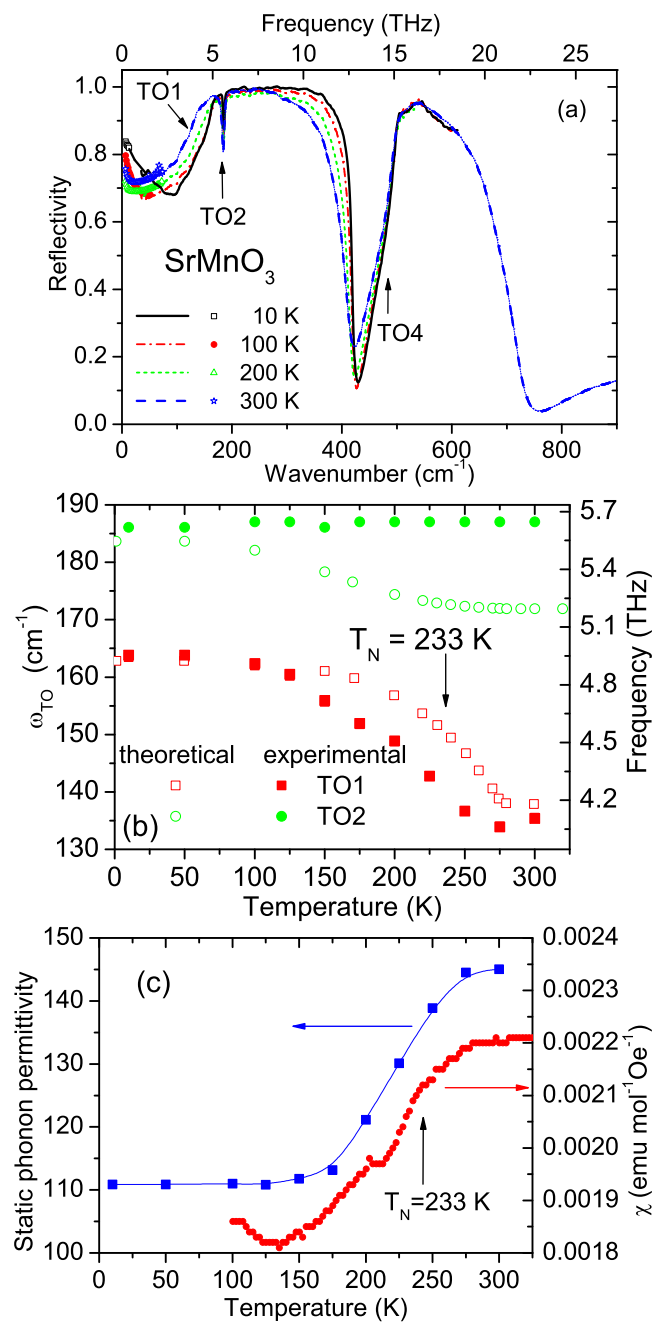

FIG. 2: (Color online) (a) Infrared reflectivity spectra at various temperatures. Frequencies of all three polar phonons are marked by arrows (TO3 is silent). (b) Temperature dependence of TO1 and TO2 phonon frequencies obtained from the fits of IR reflectivity (solid symbols) and first principles calculations (open symbols). (c) Temperature dependence of the static phonon permittivity obtained from the sum of all phonon and electronic $\left(\varepsilon_{\infty}=7.6\right)$ contributions (left scale). Temperature dependence of the static magnetic susceptibility (right scale).

\section{RESULTS}

\section{A. IR spectrocopy studies}

FIR reflectivity spectra of cubic $\mathrm{SrMnO}_{3}$ taken at various temperatures down to $10 \mathrm{~K}$ are shown in Fig. 2 $\mathrm{a}$. Three polar optical modes (symmetry $F_{1 u}$ ) typical of the cubic $\operatorname{P} m \overline{3} m$ perovskite structure are seen. The lowest-frequency TO1 phonon undergoes a remarkable temperature-induced shift of its frequency $\omega_{T O 1}$. It increases by $18 \%$ on cooling (Fig. 2b) and therefore its dielectric strength $\Delta \varepsilon_{1}$ decreases by $42 \%$ (from 124 to 87 ) 

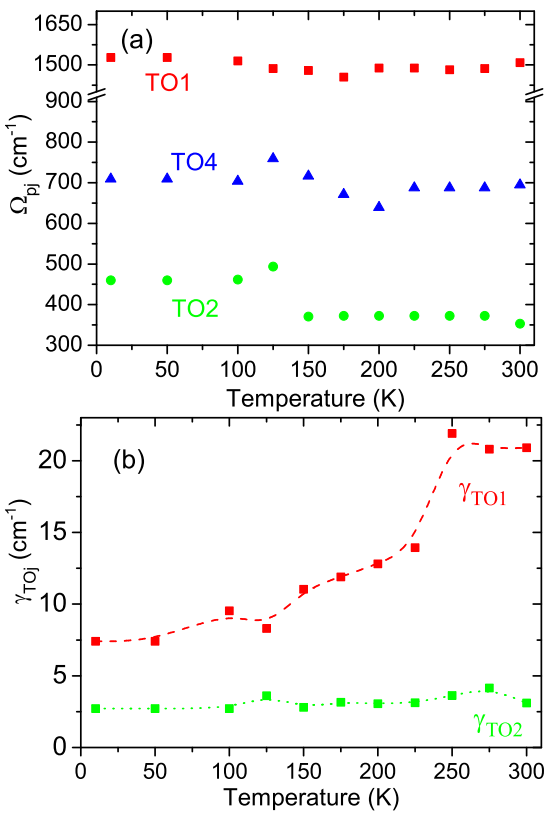

FIG. 3: (Color online) (a) Temperature dependence of experimental plasma frequencies $\Omega_{p j}=\sqrt{\Delta \varepsilon_{j}} \omega_{T O j}$ of the all polar phonons. (b) Temperature dependence of TO1 and TO2 phonon damping obtained from the fits of IR spectra. Lines are guides for eyes.

with decreasing temperature. The dielectric strength of the other two phonons is small $\left(\Delta \varepsilon_{2}+\Delta \varepsilon_{4}=16\right)$ and temperature independent; therefore the static phonon permittivity $\varepsilon(0)=\sum \Delta \varepsilon_{j}+\varepsilon_{\infty}$ exhibits decrease by $32 \%$ on cooling (see Fig. 2 r). The change in $\Delta \varepsilon_{j}$ follows from the requirement that the plasma frequency $\Omega_{p j}=\sqrt{\Delta \varepsilon_{j}} \omega_{T O j}$ of the $j$-th uncoupled phonon should be temperature independent. Indeed, examination of the spectra shows that all the plasma frequencies are temperature independent within experimental error (see Fig. [3a). Sakai et al $\stackrel{6}{\underline{6}}$ observed different behavior in $\mathrm{SrMnO}_{3}$ single crystal, namely decrease of $\Omega_{p 1}$ and simultaneous increase of $\Omega_{p 2}$. It means that the plasma frequency transfers from the TO1 to the TO2 mode, which is a signature of their mutual coupling. This mode coupling is caused by the fact that $\omega_{T O 1}=180 \mathrm{~cm}^{-1}$ and $\omega_{T O 2}=190 \mathrm{~cm}^{-1}$ are very close in single crystals at low temperatures, while in the investigated ceramics we found TO1 mode to be less stiffened $\left(\omega_{T O 1}=164 \mathrm{~cm}^{-1}\right)$ and therefore it is less coupled to TO2. Nevertheless, we observed a large enhancement of TO1 phonon damping near and above $\mathrm{T}_{N}$ (see Fig. 3b). The discrepancy of $\omega_{T O 1}$ in the ceramics and crystal can be attributed to different oxygen content in both samples. Note, we annealed the ceramics at $350{ }^{\circ} \mathrm{C}$ in air and at ambient pressure, while the crystal was annealed at $480{ }^{\circ} \mathrm{C}$ in oxygen atmosphere at pressure of $6.5 \mathrm{GPa} . \stackrel{5}{.}$

The temperature dependence of the static permittivity $\varepsilon^{\prime}(0)$ obtained from IR spectra is shown in Fig. 22. $\varepsilon^{\prime}(0)$ consists of the sum of dielectric contributions of all three polar phonons and electrons. The decrease of $\varepsilon^{\prime}(0)$ by $32 \%$ on cooling below $\mathrm{T}_{N}$ follows from Lydanne-SachsTeller relation $\frac{\varepsilon^{\prime}(0)}{\varepsilon_{\infty}}=\prod \frac{\omega_{L O j}^{2}}{\omega_{T O j}^{2}}$ due to the $\omega_{T O 1}$ stiffening on cooling (longitudinal phonon frequency $\omega_{L O j}$ is usually temperature independent). Note that the temperature change of $\varepsilon^{\prime}(0)$ is one order of magnitude larger than that found in $\mathrm{EuTiO}_{3}, \frac{15}{\stackrel{1}{w}}$ which is assumed to be a material with very strong spin-phonon coupling. Note as well that the static magnetic susceptibility $\chi$ exhibits temperature dependence similar to that of $\varepsilon^{\prime}(0)$ (see Fig. 2r). We have measured $\chi(\mathrm{T})$ up to $500 \mathrm{~K}$ and from its fit by a Curie-Wess law we have obtained the Curie-Weiss temperature $\Theta \cong-2300 \mathrm{~K}$. This gives evidence about strong magnetic correlations above $\mathrm{T}_{N}$. Similar $\chi(\mathrm{T})$ behavior was reported by Belik et al $l^{10}$

Note that $\chi(\mathrm{T})$ starts to decrease already near $270 \mathrm{~K}$ (Fig. 25), $40 \mathrm{~K}$ above $\mathrm{T}_{N}$. TO1 phonon frequency exhibits a minimum as well near $270 \mathrm{~K}$ (Fig. 2r). This can be caused by short-range magnetic correlations above $\mathrm{T}_{N}$, hints of which are also seen in the temperature dependence of the thermal conductivity $\kappa$ (see discussion in Section C.)

The experimentally observed TO1 phonon frequency and its temperature dependence correspond very well to the theoretical prediction obtained from first-principles calculations (see Fig. 2b). Our calculations show that the TO1 phonon eigenvector corresponds to a Slater-type vibration, i.e. to the $\mathrm{Mn}$-vibrations against oxygen octahedra. In Fig. 3 a we see that this mode has the highest plasma frequency $\Omega_{P 1}$, which gives experimental evidence that the TO1 phonon is the Slater mode. $\underline{16}$ The theoretical TO2 mode frequency corresponds well to the experimental value at $10 \mathrm{~K}$, but unlike the experimental mode, it softens slightly with increasing temperature and at room temperature it is $10 \%$ lower than the experimental value. However, this behavior is sensitive to the choice of lattice constant and the temperature dependence of the TO2 mode is reduced in calculations for which the chosen lattice constant is increased. Note that $\omega_{T O 2}$ is experimentally determined very precisely $\left( \pm 1 \mathrm{~cm}^{-1}\right)$, because its damping is very low (see Figs. $2 \mathrm{a}$ and $3 \mathrm{~b}$ ). A temperature independent $\omega_{\mathrm{TO}_{2}}(\mathrm{~T})$ like that shown in Fig. $2 \mathrm{~b}$ was reported by Sakai et al. $\underline{\underline{6}}$

Sacchetti et al $\frac{17}{\square}$ investigated FIR reflectivity spectra of cubic and hexagonal $\mathrm{SrMnO}_{3}$ at 100 and $300 \mathrm{~K}$ and obtained the same result as found here, but they did not present detailed temperature dependence of the phonon frequencies. Nevertheless, they have shown that $4 \mathrm{H}$ and $6 \mathrm{~L}$ hexagonal phases of $\mathrm{SrMnO}_{3}$ have 12 and 14 IR active modes, respectively: 17 We found only 3 polar phonons in the whole experimental temperature range. This confirms that our $\mathrm{SrMnO}_{3}$ ceramics is not contaminated by any detectable secondary hexagonal phase. 


\section{B. Raman spectroscopy studies}

Micro-Raman spectra were measured down to $80 \mathrm{~K}$. $\mathrm{SrMnO}_{3}$ crystallizes in the cubic $P m \overline{3} m$ structure, where the all phonons should be Raman inactive and only $3 F_{1 u}$ symmetry modes are IR active $\underline{\underline{16}}$ In spite of this fact, three broad bands are seen in the room-temperature Raman spectrum (see Fig. (4). These bands come, as they

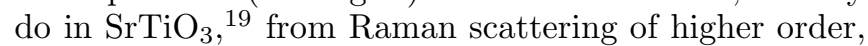
i. e. from multiphonon summation processes. The bands split on cooling, because the phonon damping decreases towards low temperatures. Several new modes also appear outside of the region of the original three bands. The experimental Raman spectrum measured at $80 \mathrm{~K}$ and its fit with assigned mode frequencies are shown in Fig. [5. The frequencies of the peaks at 192 and $508 \mathrm{~cm}^{-1}$ correspond to TO2 and TO4 frequencies (in our assignment, the TO3 mode is silent in IR and Raman spectra of perovskites with $P m \overline{3} m$ structure). However, polar TO2 and TO4 modes are forbidden in Raman spectra of the cubic structure. This means that the Raman scattering spectra give evidence of locally broken cubic structure and center of symmetry below the magnetic phase transition temperature, although our x-ray diffraction experiment performed down to $170 \mathrm{~K}$ did not reveal any change of the macroscopic symmetry. Similar magnetically induced changes of the phonon selection rules were recently reported in various transition-metal monooxides and chromium spinels ${ }^{20}$ In that work, structural investigations did not reveal any changes. However, IR spectra resolved a remarkable phonon splitting below $\mathrm{T}_{N}$. This was explained by the exchange coupling which slightly distorts the local cubic symmetry of the crystals and activates new phonons in the spectra $\underline{\underline{20}}$

There are several other new Raman bands activated below $\mathrm{T}_{N}$. The frequency of the band seen at $1017 \mathrm{~cm}^{-1}$ corresponds exactly to twice the TO4 mode frequency. A sharp band appears below $\mathrm{T}_{N}$ at $1173 \mathrm{~cm}^{-1}$. This is most probably a two magnon scattering coming from magnons at the Brillouin zone boundary. Two other strong peaks are seen near 1300 and $1500 \mathrm{~cm}^{-1}$. These come from multiphonon and/or phonon-magnon scattering. In this summation process contribute mainly phonons and magnons with maximal density of states, i.e. with wavevector from Brillouin zone boundary. Similar multiphonon and multimagnon Raman bands arising below $\mathrm{T}_{N}$ were observed in $\mathrm{BiFeO}_{3} \stackrel{21,22}{\underline{2}}$

The newly activated Raman modes cannot come from a secondary hexagonal phase, because the hexagonal phase does not have any phonons above $800 \mathrm{~cm}^{-1}$ and our low-frequency modes also do not correspond to phonons of hexagonal $\mathrm{SrMnO}_{3} \frac{17,23}{2}$ Moreover, $4 \mathrm{H}$ hexagonal $\mathrm{SrMnO}_{3}$ exhibits structural and magnetic phase transitions already around $380 \mathrm{~K}$ and $280 \mathrm{~K}$, respectively 24

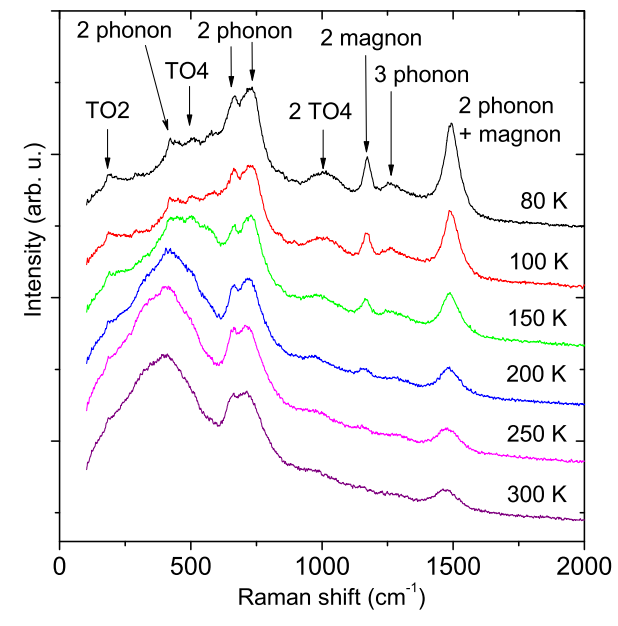

FIG. 4: (Color online) Micro-Raman spectra of $\mathrm{SrMnO}_{3}$ ceramics taken at various temperatures. The peaks seen at $80 \mathrm{~K}$ are assigned.

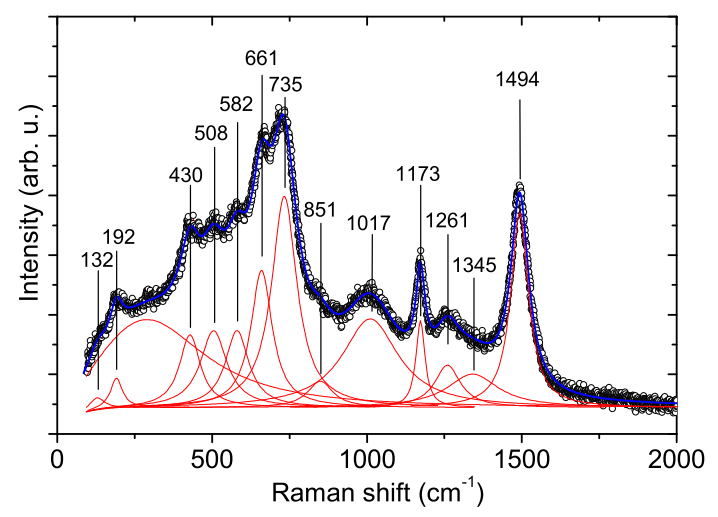

FIG. 5: (Color online) Raman scattering spectrum taken at $80 \mathrm{~K}$ and its decomposition into independent damped harmonic oscillators. Frequencies of the Raman modes are marked.

\section{Dielectric and conductivity studies}

The static permittivity $\varepsilon^{\prime}(0)$ shown in Fig. 2 r is given by phonon and electron contributions and it corresponds to $\mathrm{THz}$ experimental values measured between 100 and $300 \mathrm{~K}$. However, values of the megahertz permittivity are two orders of magnitude higher (see Fig. 6 6 ). This is caused by a non-homogeneous conductivity of the sample in the ceramic grains and grain boundaries, which is responsible for the creation of internal barrier layer capacitors on the grain boundaries. This mechanism is responsible for a "giant" effective permittivity in many dielectric or multiferroic materials $\stackrel{25-27}{-2}$

Real part of the conductivity $\sigma^{\prime}(\omega)$ exhibits strong frequency dependence typical for hopping processes $\underline{28}$ Its low-frequency part is almost frequency independent and it corresponds to a static $(D C)$ conductivity of the perco- 
lated grain boundaries. The high-frequency part of $\sigma^{\prime}(\omega)$ increases with frequency. However, it shows a plateau between 0.1 and $1 \mathrm{THz}$. Then $\sigma^{\prime}(\omega)$ again increases towards higher frequencies. The plateau $\mathrm{THz}$ conductivity value corresponds to the conductivity in the ceramic grains, while the rising $\sigma^{\prime}(\omega)$ above $1 \mathrm{THz}$ is caused by a phonon absorption.

Unfortunately, we could not study grain size dependence of the conductivity, because we could not prepare ceramics with various grain sizes. As mentioned already in section II, we had to transform the initial hexagonal ceramics to the cubic phase by 8 hour annealing at $1500{ }^{\circ} \mathrm{C}$ followed by rapid quenching of the ceramics. The grains grow at such high temperature that we could not control their size. We should mention that Sakai's single crystal was also conducting, and therefore they published only $1 \mathrm{GHz}$ permittivity at $4 \mathrm{~K}$. No radio-frequency or microwave data of $\mathrm{SrMnO}_{3}$ taken at higher temperatures were published by Sakai et al. or by other authors.

The frequency dependent conductivity $\sigma^{\prime}(\omega)$ exhibits a very interesting temperature dependence (see Fig. 6b and inset of Fig. 7). The static and low-frequency $\sigma^{\prime}(\omega)$ exhibits classical semiconductor behavior, i.e. it decreases on cooling. However, the $\mathrm{THz} \sigma^{\prime}(\omega)$ exhibits complex temperature behavior. Its value decreases on cooling from $300 \mathrm{~K}$ to $200 \mathrm{~K}$, but on further cooling it increases and at $15 \mathrm{~K}$ reaches almost one order of magnitude higher values than that at $200 \mathrm{~K}$. The former decrease of the $\mathrm{THz}$ conductivity on cooling is caused by a reduced phonon damping at low temperatures and by a corresponding lower phonon absorption in the $\mathrm{THz}$ region. However, on further cooling below $200 \mathrm{~K}$ the mean free path of charge carriers in the ceramic grains dramatically increases and therefore also the $\mathrm{THz} \sigma^{\prime}(\omega)$ increases. It can be caused by so called magnon drag effect, $\stackrel{29}{ }$ when magnons promote the high-frequency conductivity. The $\mathrm{THz}$ conductivity and permittivity spectra were possible to fit together with the IR reflectivity spectra using a sum of damped oscillators from Eq. [1] The high-frequency hopping conductivity is seen at low temperatures as a peak near $30 \mathrm{~cm}^{-1}$ in the optical conductivity $\sigma^{\prime}(\omega)$ see Fig. 8. The higher-frequency peaks correspond to the polar phonons.

In order to determine accurate chemical composition, specifically the concentration of oxygen vacancies and consequently of $\mathrm{Mn}^{3+}$ species, we have measured the thermoelectric power. Using the standard configuration entropy approach,$\frac{30}{5}$ we have determined the concentration of $\mathrm{Mn}^{3+}$ in $\mathrm{SrMn}^{4+} \mathrm{O}_{3}$ using the room temperature thermoelectric power $\left(S \sim-350 \mu \mathrm{VK}^{-1}\right)$ as $2 \%$ of all $\mathrm{Mn}^{3+}$ species. This results in the chemical formula of our ceramic sample $\mathrm{Sr}^{2+} \mathrm{Mn}_{0.98}^{4+} \mathrm{Mn}_{0.02}^{3+} \mathrm{O}_{2.99}^{2-}$. The same composition was obtained independently by measurement of the sample weight changes due to oxidation during annealing $\underline{\underline{10}}$

In addition, we have measured the temperature dependence of the thermal conductivity $\kappa$ down to $4 \mathrm{~K}$. These data are shown together with the local activa-

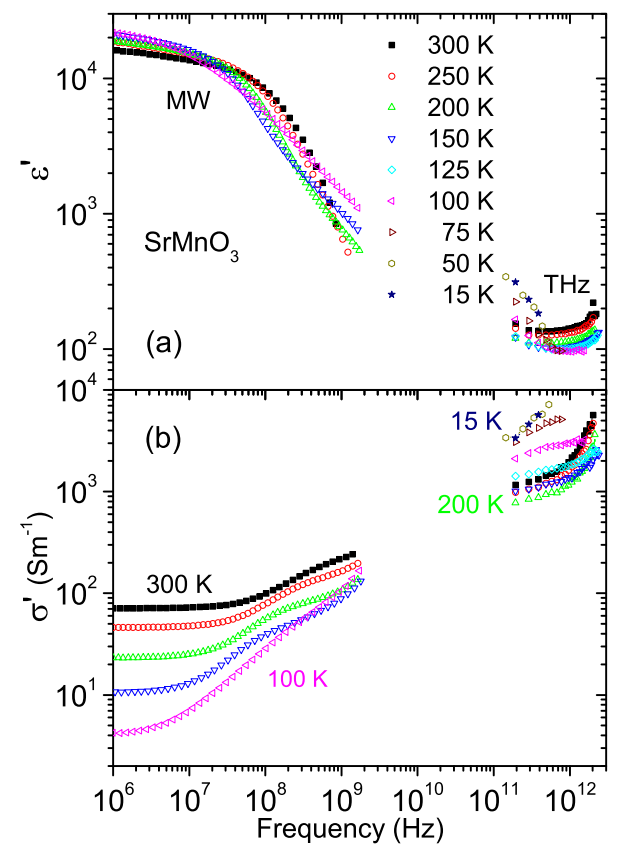

FIG. 6: (Color online) Frequency dependence of the microwave and $\mathrm{THz}$ (a) dielectric permittivity and (b) conductivity measured at various temperatures. Note that the MW data were obtained only above $100 \mathrm{~K}$.

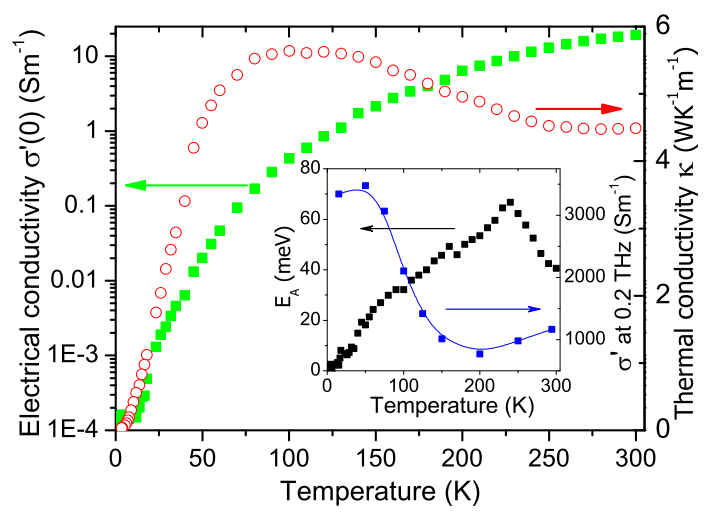

FIG. 7: (Color online) Temperature dependence of the static electrical conductivity $\sigma^{\prime}(0)$ and thermal conductivity $\kappa$. Temperature dependence of the activation energy $E_{a}$ of the $D C$ conductivity and conductivity $\sigma^{\prime}$ measured at $0.2 \mathrm{THz}$ are shown in the inset.

tion energy $E_{a}$ of $\sigma^{\prime}(0)$ in Fig. 7 Most typically, the thermal conductivity of a nonmetallic material is dominated by the phononic part, which we assumed to be true for the case of $\mathrm{SrMnO}_{3}$. The temperature dependence of $\kappa$ is then governed by the specific heat, sound velocity and phonon mean free path. These are decisively influenced by various scattering mechanisms. Considering the Debye temperature $\Theta_{D} \sim 400 \mathrm{~K}$ of $\mathrm{SrMnO}_{3}$ we anticipate the "classic" hyperbolic increase of $\kappa$ below room temperature, reflecting the dissipative Umklapp 


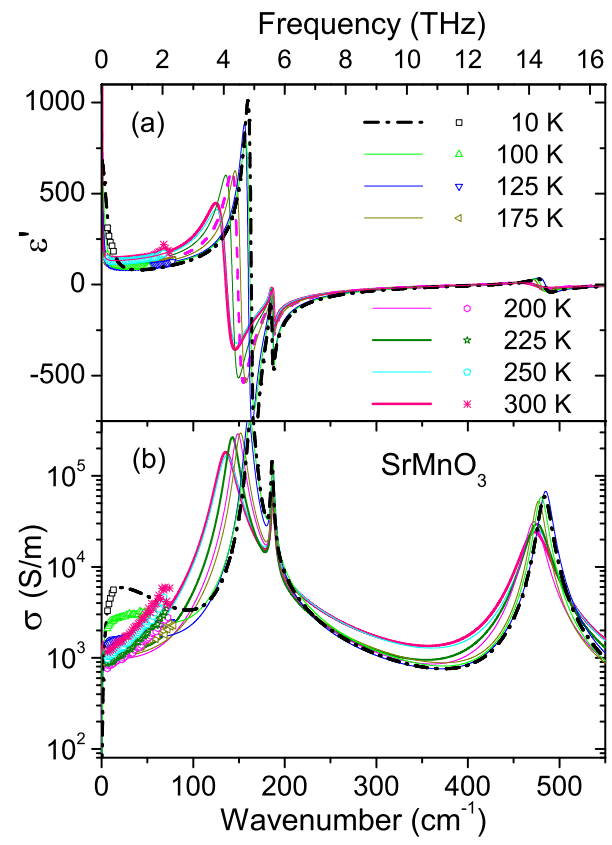

FIG. 8: (Color online) (a) Real part of the complex dielectric function and (b) optical conductivity in $\mathrm{SrMnO}_{3}$ obtained from the fits of the $\mathrm{THz}$ and IR spectra measured at various temperatures. At low frequencies and low temperatures arises a peak in the conductivity, which we fitted using highly damped oscillator.

phonon-phonon scattering. At low temperatures this is followed by the decrease critically limited by the grain boundary scattering. As we are dealing with a magnetic material, we should anticipate the phonon scattering on magnons, which should reduce $\kappa$. This is in fact observed in $\mathrm{SrMnO}_{3} ; \kappa(\mathrm{T})$ is temperature independent down to $T_{N}$ and only a very moderate increase with decreasing temperature to $100 \mathrm{~K}$ is observed. Below this temperature $\kappa$ decreases due to the decreasing specific heat. Let us note that similar behavior was recently reported by Suzuki et al. $\frac{31}{1}$ who have shown that the observed $\kappa(\mathrm{T})$ is dominated by acoustic phonons. We conclude that based on the temperature variation of $\kappa$ the phonons are critically scattered not only by magnons below $\mathrm{T}_{N}$, but also by paramagnons above $\mathrm{T}_{N}$. Contrary to the complex temperature dependence of $\kappa$, the static electrical conductivity $\sigma^{\prime}(0)$ (Fig. 7) exhibits monotonous decrease from $20 \mathrm{Sm}^{-1}$ (at $300 \mathrm{~K}$ ) down to $10^{-4} \mathrm{Sm}^{-1}$ without apparent anomalies. Nevertheless, detailed evaluation of the temperature dependence of $\sigma^{\prime}(0)$ reveals a maximum of the local activation energy $E_{a}$ near $T_{N}$, underlining the interrelation between the magnetic order and charge carrier transport. The decrease of $E_{a}(\mathrm{~T})$ to zero suggests hopping as the most likely mechanism of charge carrier transport. The fact that the $T_{N}$ is reflected by the large, broad but distinct anomaly in electric transport gives evidence that the charge, lattice and magnetic degrees of freedom are strongly coupled in $\mathrm{SrMnO}_{3}$.

\section{CONCLUSION}

First principles calculations predicted a large stiffening of TO1 phonon frequency below $\mathrm{T}_{N}$, which we experimentally confirmed. In consequence, the static phonon permittivity drops by $32 \%$. Spin-phonon coupling also influences the change of the local crystal symmetry, which allows activation of the polar phonons in Raman scattering spectra. Moreover, multiphonon and multimagnon peaks in the Raman spectra appear, sharpen and split on cooling. The $\mathrm{MHz}$ permittivity reaches giant values due to the conductivity of the ceramic grains. It comes from a small nonstoichiometry of the ceramics with chemical formula $\mathrm{Sr}^{2+} \mathrm{Mn}_{0.98}^{4+} \mathrm{Mn}_{0.02}^{3+} \mathrm{O}_{2.99}^{2-}$. The sample non-stoichiometry originates from annealing at $1500{ }^{\circ} \mathrm{C}$ during the ceramic preparation and its subsequent fast quenching to room temperature. Unfortunately, this procedure is necessary for stabilization of the cubic perovskite structure in $\mathrm{SrMnO}_{3}$, because the hexagonal structure of $\mathrm{SrMnO}_{3}$ is otherwise more stable. Sample annealing performed in air at $350{ }^{\circ} \mathrm{C}$ significantly modifies the oxygen content of the sample, which changes from $\mathrm{SrMnO}_{2.53}$ to $\mathrm{SrMnO}_{2.99}$, but unfortunately it was not possible to obtain a fully stoichiometric sample. On one hand, the static conductivity always decreases on cooling because it is influenced by the semiconducting grain boundaries. On the other hand, the mean free path of charge carriers in ceramic grains increases on cooling, and therefore also the $\mathrm{THz}$ conductivity increases with lowering temperature.

Our data show exceptionally high spin-phonon coupling in $\mathrm{SrMnO}_{3}$, which could be used for induction of the ferroelectric phase in strained thin films or doped bulk samples. Nevertheless, since good insulating behavior of the samples is necessary for possible multiferroic applications, perfect stoichiometry of $\mathrm{SrMnO}_{3}$ is required. Annealing at a high hydrostatic pressure in oxygen atmosphere could be a way to prepare highly resistive samples with strong magnetoelectric coupling.

\section{Acknowledgments}

This work was supported by the Czech Science Foundation (Projects No. P204/12/1163 and 14-14122P) and MŠMT (SIMUFER COST projects LH13048 and LD11035). The research was also partially supported by WPI Initiative (MEXT, Japan), FIRST Program of JSPS, and the Grants-in-Aid for Scientific Research (22246083) from JSPS (Japan). 
* Electronic address: kamba@fzu.cz

1 C. J. Fennie and K. M. Rabe, Phys. Rev. Lett. 97, 267602 (2006).

2 J. H. Lee, L. Fang, E. Vlahos, X. Ke, Y. W. Jung, L. F. Kourkoutis, J.-W. Kim, P. J. Ryan, T. Heeg, M. Roeckerath, et al., Nature 466, 954 (2010).

3 J. H. Lee and K. M. Rabe, Phys. Rev. Lett. 104, 207204 (2010).

4 O. Chmaissem, B. Dabrowski, S. Kolesnik, J. Mais, D. E. Brown, R. Kruk, P. Prior, B. Pyles, and J. D. Jorgensen, Phys. Rev. B 64, 134412 (2001).

${ }^{5}$ H. Sakai, J. Fujioka, T. Fukuda, D. Okuyama, D. Hashizume, F. Kagawa, H. Nakao, Y. Murakami, T. Arima, A. Q. R. Baron, et al., Phys. Rev. Lett. 107, 137601 (2011).

${ }^{6}$ H. Sakai, J. Fujioka, T. Fukuda, M. S. Bahramy, D. Okuyama, R. Arita, T. Arima, A. Q. R. Baron, Y. Taguchi, and Y. Tokura, Phys. Rev. B 86, 104407 (2012).

7 N. Hill, J. Phys. Chem. B 104, 6694 (2000).

8 J. H. Lee and K. M. Rabe, Phys. Rev. B 84, 104440 (2011).

9 T. Negas and R. S. Roth, J. Sol. State Chem. 1, 409 (1970).

10 A. A. Belik, Y. Matsushita, Y. Katsuya, M. Tanaka, T. Kolodiazhnyi, M. Isobe, and E. Takayama-Muromachi, Phys. Rev. B 84, 094438 (2011).

11 P. Kužel, H. Němec, F. Kadlec, and C. Kadlec, Optics Express 18, 15338 (2010).

12 F. Gervais, Infrared and Millimeter Waves, vol. 8 (Academic Press, 1983).

13 M. Rössle, C. N. Wang, P. Marsik, M. Yazdi-Rizi, K. W. Kim, A. Dubroka, I. Marozau, C. W. Schneider, J. Humlíek, D. Baeriswyl, et al., Phys. Rev. B 88, 104110 (2013).

14 J. Hejtmánek, E. Pollert, Z. Jirák, D. Sedmidubský, A. Strejc, A. Maignan, C. Martin, V. Hardy, R. Kužel, and Y. Tomioka, Physical Review B 66, 014426 (2002).

15 T. Katsufuji and H. Takagi, Phys. Rev. B 64, 054415 (2001).
16 J. Hlinka, J. Petzelt, S. Kamba, D. Noujni, and T. Ostapchuk, Phase Transitions 79, 41 (2006).

17 A. Sacchetti, M. Baldini, F. Crispoldi, P. Postorino, P. Dore, A. Nucara, C. Martin, and A. Maignan, Phys. Rev. B 72, 172407 (2005).

18 R. Søndenå, S. Stølen, P. Ravindran, and T. Grande, Phys. Rev. B 75, 214307 (2007).

19 C. Perry, J. H. Fertel, and T. McNelly, J. Chem. Phys. 47, 1619 (1967).

20 C. Kant, M. Schmidt, Z. Wang, F. Mayr, V. Tsurkan, J. Deisenhofer, and A. Loidl, Phys. Rev. Lett. 108, 177203 (2012).

21 M. O. Ramirez, M. Krishnamurthi, S. Denev, A. Kumar, S.-Y. Yang, Y.-H. Chu, E. Saiz, J. Seidel, A. Pyatakov, A. Bush, et al., Appl. Phys. Lett. 92, 022511 (2008).

22 M. Ramirez, A. Kumar, S. Denev, Y. Chu, J. Seidel, L. Martin, S.-Y. Yang, R. Rai, X. Xue, J. Ihlefeld, et al., Appl. Phys. Lett. 94, 161905 (2009).

23 A. Sacchetti, M. Baldini, P. Postorino, C. Martin, and A. Maignan, J. Raman Spectroscopy 37, 591 (2006).

24 A. Daoud-Aladine, C. Martin, L. C. Chapon, M. Hervieu, K. S. Knight, M. Brunelli, and P. G. Radaelli, Phys. Rev. B 75, 104417 (2007).

25 P. Lunkenheimer, V. Bobnar, A.V. Pronin, A.I. Ritus, A.A. Volkov, and A. Loidl, Phys. Rev. B 66, 052105 (2002).

26 M. Li, Z. Shen, M. Nygren, A. Feteira, D. Sinclair, and A. West, J. Appl. Phys. 106, 4106 (2009).

27 S. Kamba, D. Nuzhnyy, M. Savinov, J. Sebek, J. Petzelt, J. Prokleška, R. Haumont, and J. Kreisel, Phys. Rev. B 75, 024403 (2007).

${ }^{28}$ K. Funke, Progr. Sol. State Chem. 22, 111 (1993).

29 G. Zanmarchi and C. Haas, J. Appl. Phys. 39, 596 (1968).

30 A. Maignan, C. Martin, C. Autret, M. Hervieu, B. Raveau, and J. Hejtmanek, J. Mat. Chem. 12, 1806 (2002).

31 T. Suzuki, H. Sakai, Y. Taguchi, and Y. Tokura, J. Electronic Mat. 41, 1559 (2012). 